\title{
Frequency-dependent dielectric response model for polyimide-poly(vinilydenefluoride) multilayered dielectrics
}

\author{
Luigi Di Lillo, ${ }^{1, a)}$ Andrea Bergamini, ${ }^{2}$ Dario Albino Carnelli, ${ }^{3}$ and Paolo Ermanni ${ }^{1}$ \\ ${ }^{1}$ ETH Zurich, Centre of Structure Technologies, Leonhardstrasse 27, 8092 Zurich, Switzerland \\ ${ }^{2}$ Empa, Swiss Federal Laboratories for Materials Testing and Research, 8600 Dubendorf, Switzerland \\ ${ }^{3}$ ETH Zurich, Integrated Systems Laboratory, Gloriastrasse 35, 8092 Zurich, Switzerland
}

(Received 30 March 2012; accepted 9 June 2012; published online 3 July 2012)

A physical model for the frequency-dependent dielectric response of multilayered structures is reported. Two frequency regimes defined by the relative permittivities and volume resistivities of the layers have been analytically identified and experimentally investigated on a structure consisting of polyimide and poly(vinilydenefluoride) layers. The relative permittivity follows an effective medium model at high frequency while showing a dependence on the volume resistivity at low frequency. In this regime, relative permittivities exceeding those expected from effective medium model are recorded. These findings provide insights into inhomogeneous dielectrics behavior for the development of high energy density dielectric films. (C) 2012 American Institute of Physics. [http://dx.doi.org/10.1063/1.4731763]

The development of dielectric materials capable of storing high electric energy density is the object of considerable research efforts in the fields of power and micro electronics, energy storage, and smart mechanical structures for civilian and military applications. ${ }^{1-10}$ The energy density stored in a dielectric material $u^{\text {stor }}$, expressing also its mechanical response when subjected to an electric field, i.e., Maxwell stress ${ }^{11} \sigma$, depends linearly on its dielectric response and quadratically on its insulating properties through $\varepsilon_{r}$ and $E$, respectively, as shown in Eq. (1)

$$
u^{\text {stor }}=\frac{1}{2} \varepsilon_{0} \varepsilon_{r} E^{2}=\sigma,
$$

where $\varepsilon_{0}$ is the vacuum permittivity, $\varepsilon_{r}$ the relative permittivity, and $E$ the applied electric field. The dielectric strength of the material, $E_{\max }$, is a measure of the electron-cloud-nucleus bond, i.e., the energy band-gap, defining its volume resistivity $\rho{ }^{12}$ The $\varepsilon_{r}$ and $\rho$ are thus in inverse proportion to each other $^{13-15}$ and limit Eq. (1). The Maxwell stress is at the basis of electro-bonded laminates (EBL), structures characterized by an electrostatically tunable bending stiffness ${ }^{16}$ and damping behaviour. ${ }^{16-18}$ EBL suitable for real applications ${ }^{19,20}$ are required to exhibit values of Maxwell stress in the range of $20-30 \mathrm{MJm}^{-3}$.

By virtue of the higher dielectric strength due to barrier effects, ${ }^{21,22}$ reduced dimensionality, and consequent reduction in defects concentration, ${ }^{23}$ multilayered structures (MS) constituted by highly insulating layers spaced out with high dielectric response layers ${ }^{24}$ are considered promising material structures for high energy density applications.

An effective medium model ${ }^{25}$ is typically adopted for describing the dielectric behavior in the frequency spectrum of operation of MS. ${ }^{26}$ According to this model, the relative permittivity of a MS composed of $N$ layers is achieved by designating it as a system of $N$ capacitors connected in se-

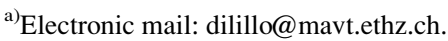

ries. Yet, this approach holds true if the dielectric material is considered to behave as an ideal capacitor, i.e., having an infinite body resistance, or if it is operated at very high frequency.

In this work, an accurate physical model accounting for the finite body resistance of the layers is detailed starting with energy conservation considerations (Eq. (2)). The equivalent series resistance (ESR), resulting from leads, terminations, and plates resistances, has not been introduced in the model since its contribution is clearly negligible in these structures.

The total energy $U(t)$ of a MS (Fig. 1(a)) can be expressed as

$$
U(t)=\sum_{i=1}^{N} \overbrace{\left(\frac{1}{2} \cdot C_{i} \cdot V_{i}^{2}(t)\right)}^{\mathrm{E}_{i}^{\text {stor }}}+\sum_{i=1}^{N} \overbrace{\left(\int_{0}^{\tau} \frac{V_{i}^{2}(t)}{R_{i}} d t\right)}^{\mathrm{E}_{\mathrm{i} i s s}} .
$$

In Eq. (2), $E_{i}^{\text {stor }}$ is the energy stored in the $i$ th layer and $E_{i}^{\text {diss }}$ the energy it dissipates over the time $\tau$. $E_{i}^{\text {diss }}$ is not considered for the analytical derivation of the relative permittivity, thus $U(t)=E_{\text {tot }}^{\text {sto }}$. The value of the voltage $V_{i}$ dropping across the $i$ th layer (Eq. (3)) is given by the weight factor of the total applied voltage $V_{T}$ with the impedances $Z_{i}$ (Eq. (4)) of the layers.

$$
V_{i}=\left|\frac{Z_{i}}{\sum_{j=1}^{N} Z_{j}}\right| V_{T}
$$

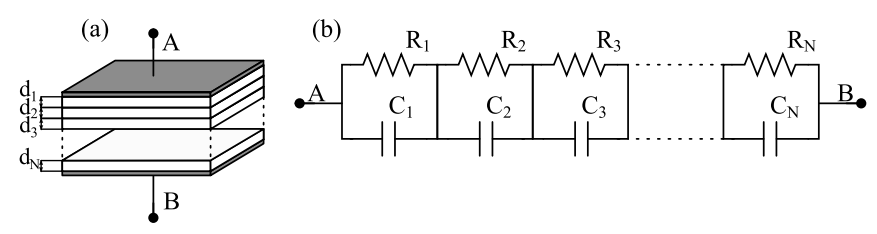

FIG. 1. In (a), a MS with the $i$ th layer having a thickness $d_{i}$. In (b), $R_{i}$ and $C_{i}$ are its body resistance and capacitance, respectively. 


$$
Z_{i}=\frac{R_{i}}{1+s C_{i} R_{i}}
$$

In Eq. (4), $R_{i}$ is the body resistance of the $i$ th layer, $s$ the complex angular frequency $s=j \omega$ with $\omega=2 \pi f$, and $C_{i}$ the capacitance of the $i$ th layer. Replacing Eq. (4) into Eq. (3) with the constitutive equations of $R_{i}$ and $C_{i}$ yields an expression for $V_{i}$ (Eq. (5)).

$$
V_{i}=\left|\frac{\rho_{i} d_{i}}{\left(1+s \varepsilon_{0} \varepsilon_{i} \rho_{i}\right) \sum_{j=1}^{N} \frac{\rho_{j} d_{j}}{1+s \varepsilon_{0} \varepsilon_{j} \rho_{j}}}\right| V_{T} .
$$

In Eq. (5), $\rho_{x}$ is the volume resistivity of the $x$ th layer, $d_{x}$ its thickness, $\varepsilon_{x}$ its relative permittivity, and $\varepsilon_{0}$ the vacuum permittivity (with $x=i, j$ ). The relative permittivity $\varepsilon_{m s}$ of a MS of thickness $d_{T}$ is obtained (Eq. (6)) by replacing Eq. (5) into Eq. (2) and expressing the total energy stored as $E_{\text {tot }}^{\text {stor }}=\frac{1}{2} C_{m s} V_{T}^{2}=\sum_{i=1}^{N} E_{i}^{\text {stor }}$, where $C_{m s}$ is the capacitance of the MS.

$$
\varepsilon_{m s}=\left(\sum_{i=1}^{N} \varepsilon_{i} d i \rho_{i}^{2}\left|\frac{1}{\left(1+s \varepsilon_{0} \varepsilon_{i} \rho_{i}\right)^{2}\left(\sum_{j=1}^{N} \frac{\rho_{j} d_{j}}{1+s \varepsilon_{0} \varepsilon_{j} \rho_{j}}\right)^{2}}\right|\right) d_{T} .
$$

The limits of Eq. (6) at low $(s \rightarrow 0)$ and high $(s \rightarrow \infty)$ frequency yield the expressions for the equivalent relative permittivity of the MS (Eq. (7)).

$$
\lim _{s \rightarrow 0} \varepsilon_{m s}=d_{T} \frac{\sum_{i=1}^{N} \varepsilon_{i} \rho_{i}^{2} d_{i}}{\left(\sum_{j=1}^{N} \rho_{j} d_{j}\right)^{2}}, \quad \lim _{s \rightarrow \infty} \varepsilon_{m s}=\frac{d_{T}}{\sum_{j=1}^{N} \frac{d_{j}}{\varepsilon_{j}}} .
$$

According to Eq. (7): (a) At low frequency, there is a direct dependence of the $\varepsilon_{m s}$ on the volume resistivity $\rho$ of the layers constituting the structure. (b) The analytical expression of $\varepsilon_{m s}$ at high frequency corresponds to the one provided by the effective medium model. (c) If the structure is composed of $N$ identical layers $\varepsilon_{m s}^{s \rightarrow 0}$ becomes $\varepsilon_{m s}^{s \rightarrow \infty}$.

Experimental evidence to the presented physical model has been brought with low frequency measurements of relative permittivity and volume resistivity on commercially available thin layers of polyimide (PI, $25 \mu \mathrm{m}$ ), supplied by $\mathrm{UBE}^{\circledR}$, Germany and Solef ${ }^{\circledR}$ poly(vinilydenefluoride) (PVDF, $375 \mu \mathrm{m}$ ), obtained from CS Hyde Company, USA. These polymers were chosen as having remarkable differences in values of $\varepsilon_{r}$ and $\rho$. MS were assembled by physically stacking layers on top of each other and making sure of their intimate contact by applying a pressure of $450 \mathrm{kPa}$ on the samples. The measurements were performed with the setup detailed by Di Lillo et al. ${ }^{27}$ They consisted in recording the current $I_{t o t}=I_{c}+I_{r}$ flowing through the MS at different voltages, from $1000 \mathrm{~V}$ to $5000 \mathrm{~V}$, within the frequency spectrum $100 \mathrm{mHz}$ to $0.5 \mathrm{mHz}$. The charging current $I_{c}(V)$ carrying knowledge of the MS's dielectric response was computed by subtracting the leakage current $I_{r}(V)$ from $I_{t o t}(V)$. The leakage current $I_{r}(V)$ was measured at the same conditions of voltages and frequency. $I_{c}(V)$ was then integrated to obtain the charge $Q(V)$ and, consequently, the relative permittivity as $\varepsilon_{r}=\frac{d_{T} Q(V)}{\varepsilon_{0} S V_{T}}$, where $S$ is the electrodes' surface. With the available setup, the lowest frequency of the signal that could be applied was limited to $f=0.5 \mathrm{mHz}$. This limit was due to the resolution of the function generator and sampling rate of the oscilloscope.

Prior to performing measurements on PI-PVDF MS, two preliminary analyses have been accomplished. The first one consisted of measurements on 30 single layers of PI and PVDF executed at all target voltages in the chosen frequency range. This step ensured that the enhanced relative permittivity, recorded at frequency $f<1 \mathrm{mHz}$ for MS with alternating different polymers, was not caused by polarization mechanisms activated in individual layers in that frequency range. The second one considered measurements on $30 \mathrm{MS}$ each consisting of the same polymers stacked in configurations of 3 to 5 on top of each other. These measurements were performed in the same frequency range and at all target voltages in order to exclude the sole geometrical effect as responsible for the $\varepsilon_{r}$ enhancement (validating point $c$ in the above list). Both the analyses provided a value of $\varepsilon_{r}$ for PI and PVDF that was constant at all voltages in the frequency spectrum investigated. Particularly, for single PI layers and multilayered PI structures, an $\varepsilon_{r}=3.5 \pm 0.2$ was recorded. As to single layers of PVDF and multilayered PVDF structures, an $\varepsilon_{r}=11.5 \pm 0.5$ was recorded. These values of $\varepsilon_{r}$ were the first input parameter for the analytical model and matched those reported in supplying companies datasheets and detailed in previous works. ${ }^{28}$ At the same time, volume resistivity $\rho$ were recorded for all the configurations, target voltages, and frequency defined above, as being the second input parameter for the analytical model.

Figure 2 displays 3 curves picturing the dielectric behavior of a MS.

Specifically, the solid blue line in Fig. 2 shows the $\varepsilon_{m s}$ behavior of a MS consisting of $\operatorname{PI}(25 \mu \mathrm{m})-\operatorname{PVDF}(375 \mu \mathrm{m})$ $\mathrm{PI}(25 \mu \mathrm{m})$ as resulting from Eq. (6). The dashed yellow line shows the behavior of $\varepsilon_{m s}$ obtained from the electrical simulation of the MS electrical equivalent (Fig. 1(b)) using the

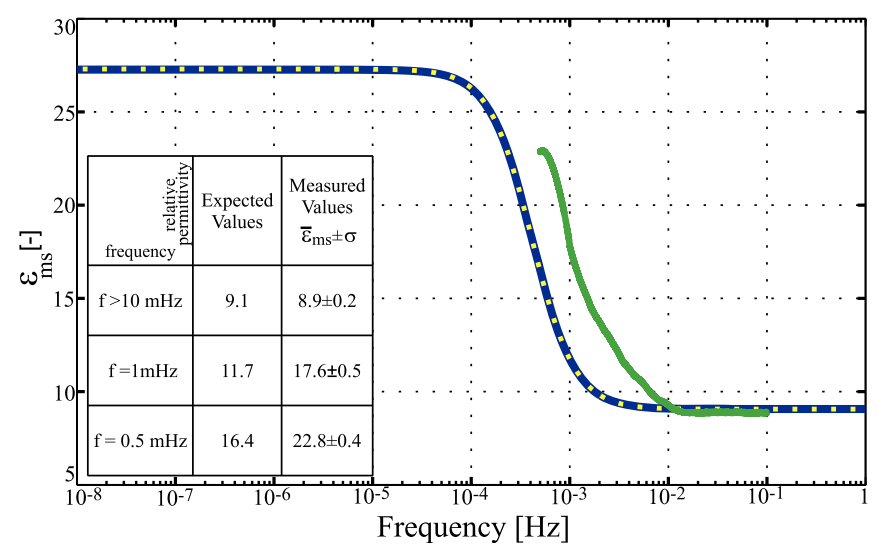

FIG. 2. Results of $\varepsilon_{m s}$ as calculated analytically (solid blue), numerically simulated (dashed yellow), and measured (green). The inset reports a comparison between expected and measured values at some target frequencies. 
same measured parameters of $\varepsilon_{r}$ and $\rho$ as in the analytical model. The electrical simulation was performed by means of Cadence ${ }^{\circledR}$ Virtuoso ${ }^{\circledR}$, a widely used software for analog circuitry design. A frequency sweep analysis in the range $10 \mathrm{nHz}-1 \mathrm{~Hz}$ yielded a frequency dependent charging current $I_{c}(f)$ which, after an accurate post-processing of its imaginary part (the one carrying information about the capacitive behavior of the system under test), led to an exactly matching result for $\varepsilon_{m s}$. The analytical and the electrical simulation results bring out the existence of two frequency domains for the relative permittivity $\varepsilon_{m s}$ of a MS. The two domains are linked by a transition region whose position and extension in frequency depend on the volume resistivities and relative permittivities of the stacked layers.

The green line in Fig. 2 reports the experimental behavior of the relative permittivity $\varepsilon_{m s}$ measured on $38 \mathrm{MS}$ with the same geometry defined in the analytical and numerical simulations. Each of them was exposed to all target voltages in the defined frequency range. Highlighted in the inset are the expected and measured values of $\varepsilon_{m s}$ at some target frequencies (high frequency domain, the one with the highest standard deviation and the lowest measured). The latter ones are expressed in the form $\varepsilon_{m s}=\bar{\varepsilon}_{m s} \pm \sigma$, where $\bar{\varepsilon}_{m s}$ is the mean value of the relative permittivity as recorded from all the measurements previously defined (the whole set of samples and target voltages) and $\sigma$ is its standard deviation. The measured values appear in good agreement with the model outcome at $f>10 \mathrm{mHz}$ where they level off at $\varepsilon_{m s}=8.9 \pm 0.2$. The proposed model is capable of representing accurately the results of the effective medium model which predicts for this MS a relative permittivity of $\varepsilon_{m s}=9.1$. The experimental curve of $\varepsilon_{m s}$ starts rising at frequencies of about $f=10.5 \mathrm{mHz}$, matching the analytical model expectation. Afterwards, it grows at a higher rate up to $f=3 \mathrm{mHz}$ leading to a value of $\varepsilon_{m s}=17.6 \pm 0.5$ (highest $\sigma$ recorded) at $f=1 \mathrm{mHz}$. At $f=0.5 \mathrm{mHz}$ the measured relative permittivity becomes $\varepsilon_{m s}=22.8 \pm 0.4$ which is higher than the values measured on single PI and PVDF layers and that is predicted by the analytical model $\left(\varepsilon_{m s}=16.4\right)$. The increase of $\varepsilon_{m s}$ at low frequency is thought to be caused mainly by an amplification of space charges at the interfaces between the layers made possible by their finite resistance which allows the charge carriers to react to the electric force caused by the applied voltage and to move through their thickness. Indeed, as confirmed from the measurements on single layers executed in the same frequency range and at same voltage conditions, the dielectric amplification is not due to the onset of polarization mechanisms occurring on single layers (constant values of relative permittivity were recorded for PI and PVDF layers). Consequently, PI-PVDF multilayer based applications can accommodate higher values of Maxwell stress (similarly, higher energy density storage capacity) at lower electric field intensity if their actuation frequency is within the low frequency domain. The onset of this domain, as shown in Eq. (7), can be tuned by changing the ratio of the volume resistivities of the layers constituting the structure (e.g., by doping with conductive nanoparticles).

Another aspect of this study concerns the consequences of the dielectric response on the voltage distribution of the
MS. Indeed, at $f<10 \mu \mathrm{Hz}$, according to Eq. (5), most of the applied voltage drops across the layers with the highest volume resistivity, PI in the proposed structure (Fig. 3). This behavior affects dramatically the maximum voltage, $V_{\text {max }}$, that the structure can withstand prior to electrical failure. It limits the capability to store high electrical energy density when the structure is operated at frequencies in the low frequency domain. Given a certain operating frequency and a certain MS, the maximum applicable voltage $V_{\text {max }}$ is defined by one of the relations introduced in Eqs. (8) and (9)

$$
\begin{aligned}
& V_{\text {max }, \text { low }}=\min \left\{E_{\text {max }, i} \times d_{i}\right\}, \\
& V_{\text {max }, \text { high }}=\min \left\{E_{\text {max }, j} \times d_{j}\right\},
\end{aligned}
$$

where $V_{\text {max, low }}$ and $V_{\text {max, high }}$ are the maximum applicable voltages at low and high frequency, respectively, $E_{\text {max }, i}$ and $E_{\text {max }, j}$ are the dielectric strengths of the layers $i$ th and $j$ th and $d_{i}$ and $d_{j}$ their thicknesses. The applicability of Eq. (8) or Eq. (9) depends on where the operating frequency is centered with respect to the domains defined by the properties of the material constituting the MS. As for the one detailed above, the capacitance of the middle layer is lower than that of the top and bottom layers, thereby at frequencies within high frequency domain, most of the voltage is dropping across its thickness. Therefore, if the thicknesses of the layers of the structure under study are $d_{j}=d_{P V D F}>d_{P I}=d_{i}$ then $V_{\text {max }, \text { high }}>V_{\text {max }, \text { low }}$ even though $E_{\text {max }, j}<E_{\text {max }, i}$ (the dielectric strength of a layer is in inverse proportion with its thickness ${ }^{29}$ ). The model therefore (a) sets the boundaries (in terms of voltages) within which the dielectric amplification effect is advantageous and (b) can help to prevent premature breakdown events due to localized electric field increase. In this work, a physical model accounting for the finite body resistance of the layers of a MS has been studied. The most significant consequences of this study are (a) when considering both dielectric and insulating properties of an inhomogeneous MS, a substantially different dielectric response than expected based on an effective medium approach is predicted; (b) It gives a basis for the prediction of the real distribution of electric field within the MS allowing for its

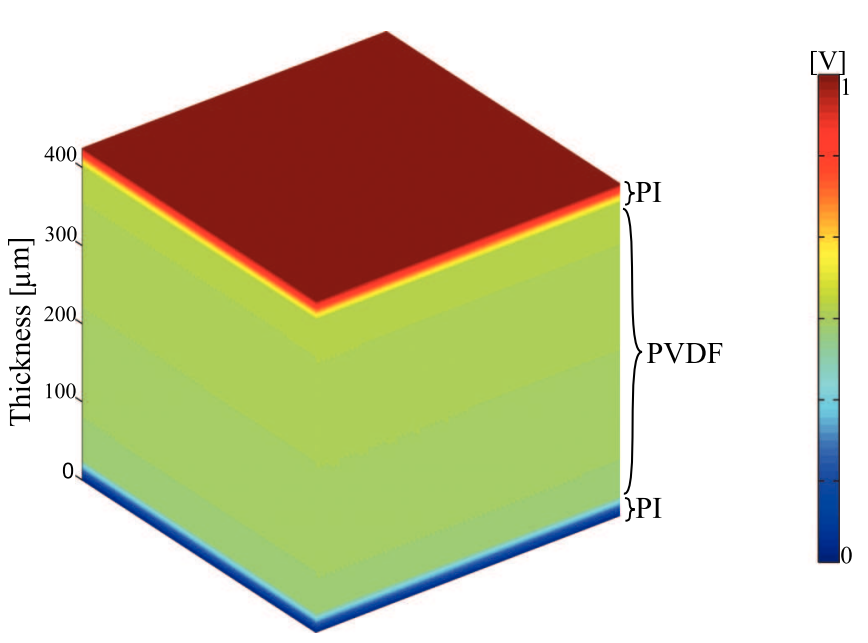

FIG. 3. Voltage distribution at frequency $f<10 \mu \mathrm{Hz}$ for the MS. The colored vertical bar shows the voltage intensity [V] normalized for the considered voltage range [0-1]. 
optimization and prediction of its failure onset. Experimental results were recorded on 38 PI-PVDF-PI structures at frequencies as low as $f=0.5 \mathrm{mHz}$ and voltages increasing from $1000 \mathrm{~V}$ to $5000 \mathrm{~V}$. The results were in good agreement with the proposed model for low frequencies and with the results predicted by the effective medium model in the high frequency domain. Structures with frequency driven operating states (e.g., EBL having a mechanical strength in shearing tuned with the frequency of the controlling signal) could be obtained. Consequences on voltage distributions allowed concluding that the enhancement in relative permittivity is counterbalanced by a decrease in the maximum applicable voltage which, when weighted on the thickness of the structure, will lead to lower values of maximum electric energy density storable. The beneficial increase of relative permittivity is limited by $V_{\text {max }, l o w}$. Following works should focus on the deviation between predicted and measured rate of rise of $\varepsilon_{m s}(f)$ and on the validation of the proposed model at even lower frequencies. Further, forced assembly coextrusion techniques ${ }^{30}$ could enable the manufacturing of much thinner and varied MS allowing for the frequency investigation of their dielectric strengths at reasonable lower electric fields.

The authors wish to thank the Swiss National Science Foundation for project funding as part of the NRP 62.

${ }^{1}$ S. Smith and P. Sen, in Power Symposium, 2008. NAPS'08. 40th North American, Calgary, Canada, 28 September 2008 (IEEE, 2008) pp. 1-6.

${ }^{2}$ K. Slenes, P. Winsor, T. Scholz, and M. Hudis, IEEE Trans. Magn. 37, 324 (2001).

${ }^{3}$ H. Wisken, F. Podeyn, and H. Weise, IEEE Trans. Magn. 37, 332 (2001).

${ }^{4}$ F. MacDougall, J. Ennis, R. Cooper, J. Bates, and K. Seal, in Pulsed Power Conference, 2003. Digest of Technical Papers. PPC-2003. 14th IEEE International, Dallas, TX, 15-18 June 2003 (IEEE, 2003), Vol. 1, pp. 513-517.

${ }^{5}$ J. Lai, S. Levy, and M. Rose, IEEE Aerosp. Electron. Syst. Mag. 7, 14 (1992).

${ }^{6} \mathrm{P}$. Ribeiro, B. Johnson, M. Crow, A. Arsoy, and Y. Liu, IEEE Proc. 89, 1744 (2001).
${ }^{7} \mathrm{H}$. Nalwa, in Low and High Dielectric Constant Materials (Academic, 1999), pp. 424-490.

${ }^{8}$ A. Aricò, P. Bruce, B. Scrosati, J. Tarascon, and W. Van Schalkwijk, Nat. Mater. 4, 366 (2005).

${ }^{9}$ P. Brochu and Q. Pei, Macromol. Rapid Commun. 31, 10 (2010).

${ }^{10}$ R. Pelrine, R. Kornbluh, Q. Pei, and J. Joseph, Science 287, 836 (2000).

${ }^{11}$ J. Stratton, Electromagnetic Theory (Wiley-IEEE, 2007), Vol. 33, pp. $83-165$.

${ }^{12}$ N. Ashcroft and N. Mermin, Solid Sate Physics (Holt Rinehart and Winston, New York, 1976).

${ }^{13}$ D. Penn, Phys. Rev. 128, 2093 (1962).

${ }^{14}$ J. McPherson, J. Kim, A. Shanware, H. Mogul, and J. Rodriguez, IEEE Trans. Electron Devices 50, 1771 (2003).

${ }^{15}$ J. McPherson, J. Kim, A. Shanware, H. Mogul, and J. Rodriguez, in Electron Devices Meeting, 2002. IEDM'02. Digest International, San Francisco, CA, 8-11 December 2002 (IEEE, 2002), pp. 633-636.

${ }^{16}$ See supplemental material at http://dx.doi.org/10.1063/1.4731763 for electro-bonded laminates characterized by an electrostatically tunable bending stiffness and damping behaviour.

${ }^{17}$ A. Bergamini, R. Christen, B. Maag, and M. Motavalli, Smart Mater. Struct. 15, 678 (2006).

${ }^{18}$ A. Bergamini, R. Christen, and M. Motavalli, Smart Mater. Struct. 16, 575 (2007).

${ }^{19}$ G. Molinari, M. Quack, V. Dmitriev, M. Morari, P. Jenny, and P. Ermanni, J. Intell. Mater. Syst. Struct. 22, 1075 (2011).

${ }^{20} \mathrm{~W}$. Raither, A. Bergamini, and P. Ermanni, in Proceedings of International Conference on Adaptive Structures Technologies ICAST, Corfu, Greece, 10-12 October 2011.

${ }^{21}$ O. Gefle, S. Lebedev, and V. Uschakov, J. Phys. D: Appl. Phys. 30, 3267 (1997).

${ }^{22}$ R. Vogelsang, T. Farr, and K. Frohlich, IEEE Trans. Dielectr. Electr. Insul. 13, 373 (2006).

${ }^{23}$ M. Chanda and S. Roy, Plastics Technology Handbook (CRC, 2006).

${ }^{24}$ M. Mackey, A. Hiltner, E. Baer, L. Flandin, M. Wolak, and J. Shirk, J. Phys. D: Appl. Phys. 42, 175304 (2009).

${ }^{25}$ D. Bergman, Phys. Rep. 43, 377 (1978).

${ }^{26}$ M. Wolak, M. Pan, A. Wan, J. Shirk, M. Mackey, A. Hiltner, E. Baer, and L. Flandin, Appl. Phys. Lett. 92, 113301 (2008).

${ }^{27}$ L. Di Lillo, A. Schmidt, D. Carnelli, P. Ermanni, G. Kovacs, E. Mazza, and A. Bergamini, J. Appl. Phys. 111, 024904 (2012).

${ }^{28}$ L. Di Lillo, D. A. Carnelli, A. Bergamini, S. Busato, and P. Ermanni, Smart Mater. Struct. 20, 057002 (2011).

${ }^{29}$ F. Forlani and N. Minnaja, Phys. Status Solidi (b) 4, 311 (1964).

${ }^{30}$ M. Ponting, T. Burt, L. Korley, J. Andrews, A. Hiltner, and E. Baer, Ind. Eng. Chem. Res. 49, 12111 (2010). 